\title{
Recombinant Vesicular Stomatitis Virus- expressing Interferon-beta and Tyrosinase Related Protein 1
}

\author{
National Cancer Institute
}

\section{Source}

National Cancer Institute. Recombinant Vesicular Stomatitis Virus-expressing Interferonbeta and Tyrosinase Related Protein 1. NCI Thesaurus. Code C157710.

A recombinant, replicating oncolytic vesicular stomatitis virus (VSV) carrying the human interferon-beta (IFN-b) gene and the tyrosinase related protein 1 (TYRP1) gene, with potential immunomodulating and antineoplastic activities. Upon intratumoral and intravenous administration, recombinant VSV-expressing IFN-b/TYRP1 preferentially replicates in tumor cells. Due to defective IFN-b-mediated innate antiviral host defense mechanisms in tumor cells, VSV is able to replicate in these cells without interference. This induces VSV-mediated cytolytic activity towards the tumor cells. By expressing human IFN-b, an IFN-b-mediated antiviral immune response in surrounding normal cells is activated which protects normal cells ag ainst virus replication and the associated VSVmediated cell lysis. Also, the IFN-b produced by VSV may activate an immune response in surrounding normal cells, involving the activation of cytotoxic T-lymphocytes (CTL), dendritic cells (DCs) and natural killer (NK) cells. This induces an anti-tumor immune response against the tumor cells. In addition, the expression of TYRP1 further activates the immune system to induce a CTL-mediated immune response against the TYRP1expressing tumor cells, thereby further killing TYRP1-expressing tumor cells. VSV, a single-stranded RNA virus belonging to the genus Vesiculovirus of the family Rhabdoviridae, is relatively nonpathogenic to healthy humans but is able to rapidly replicate in and induce apoptosis of tumor cells. TYRP1, a tumor-associated antigen (TAA), is primarily expressed in melanocytes and melanomas. 